\title{
Performance Analysis of Evolutionary Algorithms for the Minimum Label Spanning Tree Problem
}

\author{
Xinsheng Lai, Yuren Zhou, Jun He, Member, IEEE, Jun Zhang, Senior Member, IEEE
}

\begin{abstract}
A few experimental investigations have shown that evolutionary algorithms (EAs) are efficient for the minimum label spanning tree (MLST) problem. However, we know little about that in theory. In this paper, we theoretically analyze the performances of the (1+1) EA, a simple version of EA, and a simple multi-objective evolutionary algorithm called GSEMO on the MLST problem. We reveal that for the MLST $_{b}$ problem the (1+1) EA and GSEMO achieve a $\frac{b+1}{2}$-approximation ratio in expected polynomial runtime with respect to $n$ the number of nodes and $k$ the number of labels. We also find that GSEMO achieves a $(2 \ln n)$-approximation ratio for the MLST problem in expected polynomial runtime with respect to $n$ and $k$. At the same time, we show that the $(1+1)$ EA and GSEMO outperform local search algorithms on three instances of the MLST problem. We also construct an instance on which GSEMO outperforms the (1+1) EA.
\end{abstract}

Index Terms-Evolutionary algorithm; runtime complexity; approximation ratio; minimum label spanning tree; multiobjective

\section{INTRODUCTION}

The minimum label spanning tree (MLST) problem is an issue arising from practice, which seeks a spanning tree with the minimum number of labels in a connected undirected graph with labeled edges. This problem has many applications in real-life. For example, in communication networks, a communication node may communicate with other nodes via different types of channels, such as optic fiber, coaxial cable, microwave, telephone line [1]. Given a set of communication nodes, we want to find a spanning tree (a connected communication network) that uses the minimum possible number of types of channels, which can reduce the construction cost and the complexity of the network [2]. Another example is that the MLST problem has been used in data compression to increase compression rates [3], [4]. The MLST problem, proposed by Chang and Leu, has been proved to be NP-hard [2].

This work was supported in part by the National Natural Science Foundation of China $(61170081,61165003,61300044,61332002)$, in part by the EPSRC under Grant EP/I009809/1, in part by the National High-Technology Research and Development Program (863 Program) of China No. 2013AA01A212, and in part by the NSFC for Distinguished Young Scholars 61125205 . (Corresponding author: Y. Zhou).

X. Lai and Y. Zhou are with the School of Computer Science and Engineering, South China University of Technology, Guangzhou 510006, China (e-mail: yrzhou@scut.edu.cn).

$\mathrm{X}$. Lai is also with the School of Mathematics and Computer Science, Shangrao Normal University, Shangrao 334001, China.

J. He is with the Department of Computer Science, Aberystwyth University, Aberystwyth, SY23 3DB, U.K.

J. Zhang is with the Department of Computer Science, Sun Yat-Sen University, Guangzhou 510275, China, with the Key Laboratory of Digital Life, Ministry of Education, China, and also with the Key Laboratory of Software Technology, Education Department of Guangdong Province, China. Copyright (c) 2012 IEEE
For this problem, Chang and Leu have proposed two heuristic algorithms. One is the edge replacement algorithm, ERA for short, and the other is the maximum vertex covering algorithm, MVCA for short. Their experiments showed that ERA is not stable, and MVCA is very efficient.

The genetic algorithm, belonging to the larger class of EAs, is a general purpose optimization algorithm [5], [6], [7] with a strong globally searching capacity [8]. So, Xiong, Golden, and Wasil proposed a one-parameter genetic algorithm for the MLST problem. The experimental results on extensive instances generated randomly showed that the genetic algorithm outperforms MVCA [9]. Nummela and Julstrom also proposed an efficient genetic algorithm for solving the MLST problem [10].

Besides, many methods have been recently proposed for solving this NP-hard problem. Consoli and Moreno-Pérezb proposed a hybrid local search combining variable neighborhood search and simulated annealing [11]. Chwatal and Raidl presented exact methods including branch-and-cut and branch-and-cut-and-price [12]. Cerulli et al. utilized several metaheuristic methods for this problem, such as simulated annealing, reactive tabu search, the pilot method, and variable neighborhood search [13]. Consoli et al. proposed a greedy randomized adaptive search procedure and a variable neighborhood search for solving the MLST problem [14].

Since both ERA and MVCA are two original heuristic algorithms for the MLST problem, the worst performance analysis of these two algorithms, especially MVCA, is a hot research topic in recent years. Krumke and Wirth proved that MVCA has a logarithmic performance guarantee of $2 \ln n+1$, where $n$ is the number of nodes in the input graph, and presented an instance to show that ERA might perform as badly as possible [15]. Wan, Chen, and Xu further proved that MVCA has a better performance guarantee of $\ln (n-1)+1$ [16]. Xiong, Golden, and Wasil proved another bound on the worst performance of MVCA for $\mathrm{MLST}_{b}$ problems, i.e., $H_{b}=\sum_{i=1}^{b} \frac{1}{i}$, where the subscript $b$ denotes that each label appears at most $b$ times, and is also called the maximum frequency of the labels [17].

The performance of MVCA on the MLST problem has been deeply investigated. However, there is still no theoretical work which focuses on the performance analysis of EAs for the MLST problem, though a few experimental investigations have shown that EAs are efficient for this problem.

Recently, the theoretical analysis of EAs' performance on combinatorial optimization problems has received much attention. During the past few years, theoretical investigations about EAs focused on the runtime for finding globally optimal 
solutions of combinatorial optimization problems or their variants. These problems include plateaus of constant fitness [18], linear function problems [19], [20], [21], minimum cut problems [22], satisfiability problems [23], minimum spanning tree problems [24], Eulerian cycle problems [25], Euclidean traveling salesperson problems [26], the maximum matching problem [27], and so on.

Nevertheless, many combinatorial optimization problems, including the MLST problem, are NP-hard, and it is commonly believed that there is no polynomial time algorithm for them. Therefore, we usually only ask satisfying solutions to such NPhard problems in practice. Thus, we are interested in whether an approximation solution with a given satisfying quality can be efficiently obtained. In fact, the approximation performance analysis of randomized heuristics, including EAs, on NP-hard problems receives more and more attentions.

Oliveto, He, and Yao found that for minimum vertex cover problems the $(1+1)$ EA may find arbitrary bad approximation solutions on some instances, but can efficiently find the minimum cover of them by using a restart strategy [28]. Friedrich et al. proved that the $(1+1)$ EA may find almost arbitrarily bad approximation solution for minimum vertex cover problems and minimum set cover problems as well [29]. Witt proved that in the worst case the $(1+1)$ EA and the randomized local search algorithm need an expected runtime $O\left(n^{2}\right)$ to produce a $\frac{4}{3}$-approximation solution to the partition problem [30].

On the approximation performance of multi-objective EAs, Friedrich et al. revealed that the multi-objective EA efficiently finds an $\ln n$-approximation solution to the minimum set cover problem [29]. Neumann and Reichel found that multiobjective EAs can find a $k$-approximation solution for the minimum multicuts problem in expected polynomial runtime [31]. Recently, Yu, Yao, and Zhou studied the approximation performance of SEIP, a simple evolutionary algorithm with isolated population, on set cover problems. They found that SEIP can efficiently obtain an $H_{n}$-approximation solution for unbounded set cover problems, and an $\left(H_{n}-\frac{k-1}{8 k^{9}}\right)$ approximation solution for $k$-set cover problems as well [32].

Though the MLST problem is NP-hard, and the minimum spanning tree problem is in the complexity class $\mathrm{P}$, both belong to the problems of finding spanning trees with some measure to be optimized. The measure of the MLST problem is the number of labels used in the tree, whereas the measure of the minimum spanning tree problem is the total weights of the edges in the tree. The performance of EAs on the minimum spanning tree problem has been recently investigated. Neumann and Wegener showed that the $(1+1)$ EA solves the minimum spanning tree problem in expected polynomial runtime [24]. They also showed that the minimum spanning tree problem can be solved more efficiently via multi-objective optimization [33]. Another spanning tree related problem is the multiobjective minimum spanning tree problem, which is NP-hard. Recently, the performance of EAs on this NP-hard problem has also been theoretically investigated [34], [35]. However, there is no theoretical work focusing on EAs' performance for the MLST problem.

In this paper, we concentrate on the performance analysis of the $(1+1)$ EA and GSEMO (a simple multi-objective evolutionary algorithm with bit-wise mutation) for the MLST problem. We analyze the approximation performances of the $(1+1)$ EA and GSEMO on the MLST problem. For the $\mathrm{MLST}_{b}$ problem, We prove that the $(1+1)$ EA and GSEMO are $\frac{b+1}{2}$ approximation algorithms. We also reveal that GSEMO can efficiently achieve a $(2 \ln n)$-approximation ratio for the MLST problem. Though the MLST problem is NP-hard, we show that on three instances the $(1+1)$ EA and GSEMO efficiently find their global optima, while local search algorithms may be trapped in local optima. Meanwhile, we construct an additional instance where GSEMO outperforms the $(1+1)$ EA.

The rest of this paper is organized as follows. The next section describes the MLST problem, and the algorithms considered in this paper. Section III analyzes the approximation performances of the $(1+1)$ EA and GSEMO on the MLST problem, while Section IV investigates the performances of the $(1+1)$ EA and GSEMO on four instances. Finally, Section $\mathrm{V}$ concludes this paper and gives possible directions for further research.

\section{THE MLST PROBLEM AND ALGORITHMS}

First of all, we give the concepts of spanning subgraph, the MLST and $\mathrm{MLST}_{b}$ problems.

Definition 1: (Spanning subgraph) Let $G=(V, E)$ and $H=\left(V^{\prime}, E^{\prime}\right)$ be two graphs, where $V\left(V^{\prime}\right)$ is the set of nodes of $G(H)$, and $E\left(E^{\prime}\right)$ is the set of edges of $G(H)$. If $V^{\prime}=V$ and $E^{\prime} \subseteq E$, then $H$ is a spanning subgraph of $G$.

Definition 2: (The MLST problem) Let $G=(V, E, L)$ be a connected undirected graph, where $V, E$, and $L=$ $\{1,2, \ldots, k\}$ are respectively the sets of $n$ nodes, $m$ edges, and $k$ labels. Each edge associates with a label by a surjective function $l: E \rightarrow L$. The MLST problem is to seek a spanning tree with the minimum number of labels in the input graph $G$.

From the definition of the MLST problem, it is easy to see that each edge $e \in E$ has a unique label $l(e) \in L$, and each label in $L$ has at least one edge associating with it.

Definition 3: (The MLST $_{b}$ problem) For an MLST problem, if each label appears at most $b$ times, then it is an $\mathrm{MLST}_{b}$ problem.

Clearly, the $\mathrm{MLST}_{b}$ problem is a special case of the MLST problem.

Our goal in this paper is to seek a connected spanning subgraph with the minimum number of labels rather than a spanning tree with the minimum number of labels, since any spanning tree contained in such a spanning subgraph is an MLST. This is an alternative formulation of the MLST problem which has been adopted in papers [9], [10].

Since each edge has exactly one label, if a label is selected then all edges with such a label have been selected. Therefore, we aim to find a set of selected labels such that the edges with these labels construct a spanning subgraph of the input graph and the number of selected labels is minimized. Thus, a set of selected labels is a solution.

Further, we encode a solution as a bit string $X=$ $\left(x_{1}, \ldots, x_{k}\right)$ which has been used in [9], where $k=|L|$ and bit $x_{i} \in\{0,1\}$ corresponding to label $i$. If $x_{i}=1(i=$ $1,2, \ldots, k)$, then label $i$ is selected, otherwise it is not. Thus, 
a bit string $X$ represents a label subset, and $|X|$ represents the number of labels contained in $X$.

We consider the spanning subgraph $H(X)$ of $G$, where $H(X)$ is a spanning subgraph restricted to edges with labels that the corresponding bits in $X$ are set to 1 . We call a solution $X$ such that $H(X)$ is a connected spanning subgraph a feasible solution. A feasible solution with the minimum number of labels is a globally optimal solution.

For solving the MLST problem, the $(1+1)$ EA uses a fitness function. The fitness function is defined as

$$
f i t(X)=(c(H(X))-1) * k^{2}+|X|,
$$

where $c(H(X))$ is the number of connected components in $H(X), k$ is the total number of labels in $L$, and $|X|=$ $\sum_{i=1}^{k} x_{i}$, i.e., the number of labels contained in $X$.

The fitness function should be minimized. The first target is to make sure that $H(X)$ is a connected spanning subgraph, and the second target is to make sure that the number of labels in the connected spanning subgraph is minimized.

Recall that a feasible solution $X$ satisfies that $H(X)$ is a connected spanning subgraph, i.e., $c(H(X))=1$. Therefore, the fitness value of a feasible solution equals to the number of labels contained in it.

We also define the fitness vector for GSEMO as a vector $(c(H(X),|X|)$, where $c(H(X))$ and $|X|$ are simultaneously minimized by GSEMO.

The following algorithms are those considered in this paper.

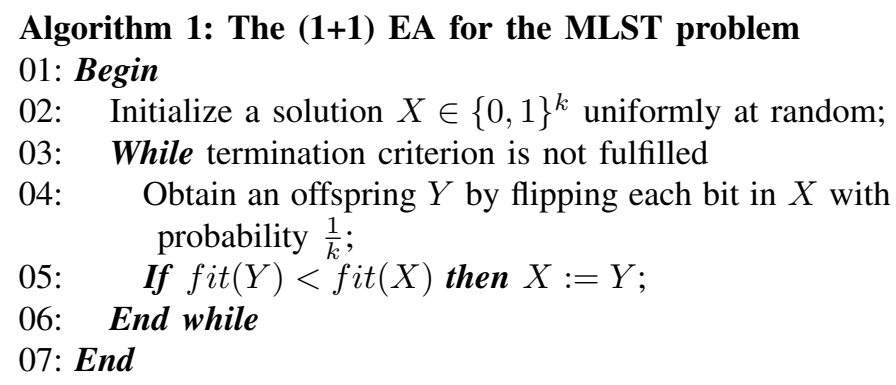

The $(1+1)$ EA starts with an arbitrary solution, and repeatedly uses mutation operator to generate an offspring solution from the current one. If the offspring solution is strictly better than the current one, then the $(1+1)$ EA uses it to replace the current solution.

Another algorithm for the MLST problem is the local search algorithm with the 2-switch neighborhood (LS2N), which is proposed by Brüggemanna, Monnot, and Woeginger. We now describe some concepts about it.

We use $X_{1}-X_{2}$ to denote the set of labels that are contained in $X_{1}$ but not in $X_{2}$, where $X_{1}$ and $X_{2}$ are two solutions. For example, if $X_{1}=\{1,2,3,4,5\}$ and $X_{2}=\{1,2,3,6\}$, then we have $X_{1}-X_{2}=\{4,5\}$ and $X_{2}-X_{1}=\{6\}$. Thus, we have $\left|X_{1}-X_{2}\right|=2$ and $\left|X_{2}-X_{1}\right|=1$.

Definition 4: [36]( $h$-switch neighborhood) Let $h \geq 1$ be an integer, and let $X_{1}$ and $X_{2}$ be two feasible solutions for some instance of the MLST problem. We say that $X_{2}$ is in $h$-switch neighborhood of $X_{1}$, denoted by $X_{2} \in h$ $\operatorname{SWITCH}\left(X_{1}\right)$, if and only if

$$
\left|X_{1}-X_{2}\right| \leq h \text { and }\left|X_{2}-X_{1}\right| \leq h .
$$

In other words, $X_{2} \in h$-SWITCH $\left(X_{1}\right)$ means that $X_{2}$ can be derived from $X_{1}$ by first removing at most $h$ labels from $X_{1}$ and then adding at most $h$ labels to it.

LS2N: in algorithm 1, if the initial solution $X$ is an arbitrary feasible solution, and the offspring $Y$ is selected from the 2switch neighborhood of $X$, then it is LS2N [36].

GSEMO has been investigated on covering problems [29], minimum spanning tree problems [33], [34], [35], and also pseudo-Boolean functions [37], [38]. GSEMO for the MLST problem is described as follows.

\section{Algorithm 2: GSEMO for the MLST problem 01: Begin}

02: Initialize a solution $X \in\{0,1\}^{k}$ uniformly at random; 03: $\quad P \leftarrow\{X\}$;

04: While termination criterion is not fulfilled

05: $\quad$ Choose a solution $X$ from $P$ uniformly at random;

06: $\quad$ Obtain an offspring $Y$ by flipping each bit in $X$ with probability $\frac{1}{k}$;

07: If $Y$ is not dominated by $\forall X \in P$ then

08: $Q:=\{X \mid X \in P$, and $Y$ dominates $X\}$;

09: $\quad P \leftarrow P \cup\{Y\} \backslash Q$;

10: $\quad$ End if

11: End while

12: End

In algorithm 2, $P$ is a population used to preserve those solutions which cannot be dominated by any other from the population. The concept of dominance is defined as follows.

Suppose that the fitness vectors of solutions $X$ and $Y$ are $(c(H(X)),|X|)$ and $(c(H(Y)),|Y|)$, respectively. We say that $X$ dominates $Y$, if one of the following two conditions is satisfied:

(1) $c(H(X))<c(H(Y))$ and $|X| \leq|Y|$;

(2) $c(H(X)) \leq c(H(Y))$ and $|X|<|Y|$.

For the sake of completeness, we describe another two algorithms in the following, which are greedy algorithms.

The first one is the modified MVCA. It starts with a solution containing no labels, and each time selects a label such that when this label is chosen the decrease in the number of connected components is the largest.

\section{Algorithm 3: The modified MVCA [17]}

Input: A given connected undirected graph $G=(V, E, L)$, $L=\{1, \ldots, k\}$.

01: $\quad$ Let $C$ be the set of used labels, $C:=\emptyset$;

02: Repeat

03: Let $H$ be the spanning subgraph of $G$ restricted to edges with labels from $C$;

04: $\quad$ For all $i \in L \backslash C$ do

05: $\quad$ Determine the number of connected components when inserting all edges labeled by $i$ in $H$;

\section{6: $\quad$ End for}

07: $\quad$ Choose label $i$ with the smallest resulting number of connected components: $C:=C \cup\{i\}$;

08: Until $H$ is connected.

Output: $H$ 
The second is called in this paper the modified MVCA with contraction, which has been investigated in [15].

The modified MVCA with contraction: in algorithm 3, if we contract each connected component in $H$ to a supernode after step 3, then we obtain the modified MVCA with contraction.

\section{APPROXIMATION PERFormANCES OF THE $(1+1)$ EA AND GSEMO ON THE MLST PROBLEM}

The following is the concept of approximation ratio (solution). Given a minimization problem $R$ and an algorithm $\mathrm{A}$, if for an instance $I$ of $R$, the value of the best solution obtained in polynomial runtime by $\mathrm{A}$ is $\mathrm{A}(I)$, and $\max _{I \in R} \frac{\mathrm{A}(I)}{\mathrm{OPT}(I)}=r$, where $\mathrm{OPT}(I)$ is the value of the optimal solution of $I$, then we say that $\mathrm{A}$ achieves an $r$-approximation ratio (solution) for $R$.

Although the MLST problem is NP-hard, we reveal that the $(1+1)$ EA and GSEMO guarantee to achieve an approximation ratio for the $\mathrm{MLST}_{b}$ problem in expected polynomial runtime with respect to $n$ the number of nodes and $k$ the number of labels, and that GSEMO guarantees to obtain an approximation ratio for the MLST problem in expected polynomial runtime with respect to $n$ and $k$.

\section{A. The approximation guarantees of the $(1+1) E A$ and GSE- $M O$ on the $M L S T_{b}$ problem}

In this subsection, let $G=(V, E, L)$ be an arbitrary instance of the $\mathrm{MLST}_{b}$ problem, where $|V|=n,|L|=k$, and $b \geq 2$, and let $\operatorname{OPT}(G)$ denote the number of labels used in the global optimum of $G$.

We show that the $(1+1)$ EA and GSEMO guarantee to achieve a $\frac{b+1}{2}$-approximation ratio for $G$ in expected polynomial runtime with respect to $n$ and $k$ in two steps. Since $G$ is an arbitrary instance of the $\mathrm{MLST}_{b}$ problem, we reveal that the $(1+1)$ EA and GSEMO guarantee to achieve a $\frac{b+1}{2}$ approximation ratio for the $\mathrm{MLST}_{b}$ problem in expected runtime polynomial in $n$ and $k$. First, we prove that the $(1+1)$ EA and GSEMO starting with any initial solution find a feasible solution for $G$ in expected runtime polynomial in $n$ and $k$, then prove that starting with any feasible solution the $(1+1)$ EA and GSEMO find a $\frac{b+1}{2}$-approximation solution for $G$ in expected polynomial runtime with respect to $k$ by simulating the result proved in [36].

We now prove that starting with any initial solution, the $(1+1)$ EA can efficiently find a feasible solution of $G$.

Lemma 1: The $(1+1)$ EA starting with any initial solution finds a feasible solution for $G$ in expected runtime $O(n k)$.

Proof: According to the fitness function (1), during the optimization process of the $(1+1) \mathrm{EA}$, the number of connected components will never be increased.

Let $X$ be the current solution of $G$. If $X$ is not a feasible solution, then the number of connected components of the spanning subgraph $H(X)$ is greater than one. Note that $G$ is connected. There must exist a label such that when it is added to $X$ the number of connected components will be decreased by at least one. The probability of adding this label to $X$ is $\frac{1}{k}\left(1-\frac{1}{k}\right)^{k-1} \geq \frac{1}{e k}$, which implies that in expected runtime
$O(k)$ the number of connected components will be decreased by at least one.

Since there are at most $n$ connected components, a feasible solution of $G$ will be found by the $(1+1)$ EA starting with any initial solution in expected runtime $O(n k)$.

Brüggemann, Monnot, and Woeginger have proved that L$\mathrm{S} 2 \mathrm{~N}$ is a $\frac{b+1}{2}$-approximation algorithm for the $\mathrm{MLST}_{b}$ problem [36], so we have the following lemma.

Lemma 2: LS2N can find a feasible solution for $G$ with at $\operatorname{most} \operatorname{OPT}(G) \cdot \frac{b+1}{2}$ labels.

We partition all feasible solutions of $G$ into two disjoint sets. One is $S_{1}=\left\{X \mid X \in\{0,1\}^{k}, X\right.$ is a feasible solution of $G$, $\left.|X| \leq \operatorname{OPT}(G) \cdot \frac{b+1}{2}\right\}$, and the other is $S_{2}=\left\{X \mid X \in\{0,1\}^{k}\right.$, $X$ is a feasible solution of $\left.G,|X|>\operatorname{OPT}(G) \cdot \frac{b+1}{2}\right\}$.

From Lemma 2, we derive a property regarding the 2 -switch neighborhood.

Corollary 1: If $X$ is a feasible solution of $G$, and $X \in$ $S_{2}$, then there must exist a feasible solution $X^{\prime} \in 2$ $S W \operatorname{ITCH}(X)$ whose fitness value is 1 or 2 less than that of $X$.

Next, we will show that starting with an arbitrary feasible solution, the $(1+1)$ EA can efficiently find a $\frac{b+1}{2}$ approximation solution of $G$.

Lemma 3: For $G$, the $(1+1)$ EA starting with an arbitrary feasible solution finds a $\frac{b+1}{2}$-approximation solution in expected runtime $O\left(k^{4}\right)$.

Proof: Let $X$ be the current feasible solution of $G$. By Corollary 1 , if $X \in S_{2}$, then there must exist a feasible solution $X^{\prime} \in 2-S W \operatorname{ITCH}(X)$ whose fitness value is 1 or 2 less than that of $X$. So, replacing $X$ with $X^{\prime}$ decreases the fitness value by at least 1 . Since a feasible solution belonging to $S_{2}$ has at most $k$ labels, then after at most $k-\mathrm{OPT}(G) \cdot \frac{b+1}{2}$ such replacing steps a feasible solution belonging to $S_{1}$ will be found.

Now, we calculate the expected runtime for the $(1+1)$ EA to find $X^{\prime}$. Since $X^{\prime} \in 2-S W I T C H(X)$ and $\left|X^{\prime}\right|<|X|$. There exist three cases. The first is that $X^{\prime}$ is obtained by removing one exact label from $X$. The second is that $X^{\prime}$ is obtained by removing two exact labels from $X$. The third is that $X^{\prime}$ is obtained by removing two exact labels from $X$ and adding one exact label to it.

Obviously, the worst case is the third one, since in this case three bits of $X$ must be simultaneously flipped by the $(1+1)$ EA. In this case, the probability that the $(1+1)$ EA finds $X^{\prime}$ is $\frac{1}{k^{3}}\left(1-\frac{1}{k}\right)^{k-3} \geq \frac{1}{e k^{3}}$. So, the expected runtime for the $(1+1)$ EA to find a feasible solution $X^{\prime} \in 2-S W I T C H(X)$ is $O\left(k^{3}\right)$, which means that the expected runtime for the $(1+1)$ EA to reduce the fitness value by at least one is $O\left(k^{3}\right)$.

Therefore, the expected runtime for the $(1+1)$ EA starting with an arbitrary feasible solution to find a $\frac{b+1}{2}$-approximation solution for $G$ is $O\left(\left(k-\operatorname{OPT}(G) \cdot \frac{b+1}{2}\right) k^{3}\right)=O\left(k^{4}\right)$, as $\operatorname{OPT}(G) \cdot \frac{b+1}{2} \leq k$.

Combining Lemmas 1 and 3, and noting that $G$ is an arbitrary instance of the $\mathrm{MLST}_{b}$ problem, we obtain the following theorem.

Theorem 1: The (1+1) EA starting with any initial solution finds a $\frac{b+1}{2}$-approximation solution for the $\mathrm{MLST}_{b}$ problem in expected runtime $O\left(n k+k^{4}\right)$. 
As we will see below, GSEMO can also efficiently obtain such an approximation solution for the $\mathrm{MLST}_{b}$ problem.

Theorem 2: GSEMO starting with any initial solution finds a $\frac{b+1}{2}$-approximation solution for the $\mathrm{MLST}_{b}$ problem in expected runtime $O\left(n k^{2}+k^{5}\right)$.

Proof: For GSEMO, the fitness vector of any solution $X$ is $(c(H(X)),|X|)$. For a given value $d$ of $|X|$, there is at most one solution in the population whose fitness vector is $(*, d)$, so the population size is $O(k)$ as $|X|$ takes value from $\{0,1, \ldots, k\}$, where $k$ is the number of labels contained in the label set.

Consider the arbitrary instance $G$, and let $X$ be the solution among the population such that $c(H(X))$ is the minimum. If $c(H(X))>1$, then there exists a label $l$ such that when it is added the number of connected components will be reduced by at least one, as $G$ is connected. The probability of selecting $X$ from the population is $\Omega\left(\frac{1}{k}\right)$, as the population size is $O(k)$, and the probability of flipping only the bit corresponding to label $l$ is $\frac{1}{k}\left(1-\frac{1}{k}\right)^{k-1}=\Omega\left(\frac{1}{k}\right)$, so a solution $X^{\prime}$ such that $c\left(H\left(X^{\prime}\right)\right)$ is at least one less than $c(H(X))$ will be included in the population in expected runtime $O\left(k^{2}\right)$.

Since there are at most $n$ connected components in the spanning subgraph induced by any solution, a feasible solution will be included in the population in expected runtime $O\left(n k^{2}\right)$.

At least one feasible solution is now included in the population. Let $X$ be the feasible solution which has the minimum labels among all feasible solutions in the population. If the number of labels contained in $X$ is greater than $\frac{b+1}{2} \cdot \operatorname{OPT}(G)$, then according to Corollary 1 there exists a feasible solution $X^{\prime} \in 2-S W I T C H(X)$ such that $\left|X^{\prime}\right|$ is at least one less than $|X|$. According to the proof of Lemma 3, the expected runtime to generate such a solution $X^{\prime}$ from $X$ is $O\left(k^{3}\right)$. Since the expected runtime to select solution $X$ from the population is $O(k)$, such a solution $X^{\prime}$ will be included in the population in expected runtime $O\left(k^{4}\right)$.

Therefore, a $\frac{b+1}{2}$-approximation solution of $G$ will be included in the population in expected runtime $O\left(\left(k-\frac{b+1}{2}\right.\right.$. $\left.\mathrm{OPT}(G)) k^{4}\right)=O\left(k^{5}\right)$ once a feasible solution is found.

Hence, GSEMO starting with any initial solution will find a $\frac{b+1}{2}$-approximation solution for $G$ in expected runtime $O\left(n k^{2}+k^{5}\right)$. Noting that $G$ is an arbitrary instance of the $\mathrm{MLST}_{b}$ problem, we obtain the theorem.

\section{B. The approximation guarantee of GSEMO on the MLST problem}

In this subsection, let $G^{\prime}=\left(V^{\prime}, E^{\prime}, L^{\prime}\right)$ be an arbitrary instance of the MLST problem, where $\left|V^{\prime}\right|=n$ and $\left|L^{\prime}\right|=k$, and let $\operatorname{OPT}\left(G^{\prime}\right)$ be the number of labels used in the minimum label spanning tree $T^{*}$ of $G^{\prime}$.

Now we analyze the approximation guarantee of GSEMO on the MLST problem by simulating the modified MVCA with contraction. Similar to Lemma 2 in [15], we have the following lemma.

Lemma 4: For $G^{\prime}$, if $n \geq 2$, then there exists a label such that the number of connected components of the spanning subgraph restricted to edges with this label is not more than $\left\lfloor n\left(1-\frac{1}{2 \mathrm{OPT}\left(G^{\prime}\right)}\right)\right\rfloor$.
Proof: Note that $G^{\prime}$ is a connected undirected graph. Since the minimum label spanning tree $T^{*}$ has exactly $n-1$ edges, there must exist a label, say $j$, such that the number of edges in $T^{*}$ labeled by $j$ is at least $\left\lceil\frac{n-1}{\operatorname{OPT}\left(G^{\prime}\right)}\right\rceil$. Hence, the number of connected components of the spanning subgraph, restricted to edges with label $j$, is not more than $n-\left\lceil\frac{n-1}{\operatorname{OPT}\left(G^{\prime}\right)}\right\rceil=\left\lfloor n\left(1-\frac{1}{\mathrm{OPT}\left(G^{\prime}\right)}\right)+\frac{1}{\mathrm{OPT}\left(G^{\prime}\right)}\right\rfloor$. When $n \geq 2$, we have $\left\lfloor n\left(1-\frac{1}{\mathrm{OPT}\left(G^{\prime}\right)}\right)+\frac{1}{\mathrm{OPT}\left(G^{\prime}\right)}\right\rfloor \leq\left\lfloor n\left(1-\frac{1}{2 \mathrm{OPT}\left(G^{\prime}\right)}\right)\right\rfloor$.

Further, for a spanning subgraph $H(X)$ of $G^{\prime}$, we have the following corollary.

Corollary 2: Let $s$ be the number of connected components of $H(X)$. If $s \geq 2$, then there is a label such that when it is added to $X$ the number of connected components will be reduced to not more than $\left\lfloor s\left(1-\frac{1}{2 \mathrm{OPT}\left(G^{\prime}\right)}\right)\right\rfloor$.

Proof: Contracting each connected component of $H(X)$ to a supernode, then $G^{\prime}$ is converted to $G^{\prime \prime}$ with $s$ nodes. Suppose that the number of labels used in the minimum label spanning tree of $G^{\prime \prime}$ is $\operatorname{OPT}\left(G^{\prime \prime}\right)$. According to Lemma 4, there is a label in $G^{\prime \prime}$ such that the number of connected components of the spanning subgraph, restricted to edges with this label, is not more than $\left\lfloor s\left(1-\frac{1}{2 \mathrm{OPT}\left(G^{\prime \prime}\right)}\right)\right\rfloor$. Noting that the number of labels used in the minimum label spanning tree of $G^{\prime}$ is $\operatorname{OPT}\left(G^{\prime}\right)$, it is clear that $\mathrm{OPT}\left(G^{\prime \prime}\right) \leq \mathrm{OPT}\left(G^{\prime}\right)$. Thus, $\left\lfloor s\left(1-\frac{1}{2 \mathrm{OPT}\left(G^{\prime \prime}\right)}\right)\right\rfloor<\left\lfloor s\left(1-\frac{1}{2 \mathrm{OPT}\left(G^{\prime}\right)}\right)\right\rfloor$. In other words, there is a label such that when it is added to $X$ the number of connected components of $H(X)$ will be reduced to not more than $\left\lfloor s\left(1-\frac{1}{2 \mathrm{OPT}\left(G^{\prime}\right)}\right)\right\rfloor$.

Based on Corollary 2, we prove that GSEMO guarantees to find a $(2 \ln n)$-approximate solution for the MLST problem in expected polynomial runtime with respect to $n$ and $k$.

Theorem 3: GSEMO starting with any initial solution finds a $(2 \ln n)$-approximation solution for the MLST problem in expected runtime $O\left(k^{2} \ln k+k^{3} \ln n\right)$.

Proof: Consider the arbitrary instance $G^{\prime}$ of the MLST problem.

We first reveal that GSEMO starting with any initial solution will find the all-zeros bit string for $G^{\prime}$ in expected runtime $O\left(k^{2} \ln k\right)$, then reveal that GSEMO finds a $(2 \ln n)$ approximation solution for $G^{\prime}$ in expected runtime $O\left(k^{3} \ln n\right)$ after the all-zeros bit string being included in the population. Combining them, and noting that $G^{\prime}$ is an arbitrary instance of the MLST problem, we obtain the theorem.

We now investigate the expected runtime that GSEMO starting with any initial solution finds the all-zeros bit string with Pareto optimal fitness vector $(n, 0)$. Once it is found, it can never be removed from the population. If it is not included in the population, then GSEMO can choose a solution $X$ from $P$ which contains the minimum number of labels among all solutions in the population with probability $\Omega\left(\frac{1}{k}\right)$, as the population size is $O(k)$. The event of flipping one of $|X|$ bits whose value is 1 will decrease the number of labels, and the probability of this event is $\left(\begin{array}{c}|X| \\ 1\end{array}\right) \frac{1}{k}\left(1-\frac{1}{k}\right)^{k-1} \geq \frac{|X|}{e k}$. So, the expected runtime that GSEMO includes a solution which contains $|X|-1$ labels is $O\left(\frac{k^{2}}{|X|}\right)$. Following this way, the allzeros bit string will be included in the population in expected runtime $O\left(\sum_{i=|X|}^{1} \frac{k^{2}}{i}\right)=O\left(k^{2} \ln |X|\right)=O\left(k^{2} \ln k\right)$. 


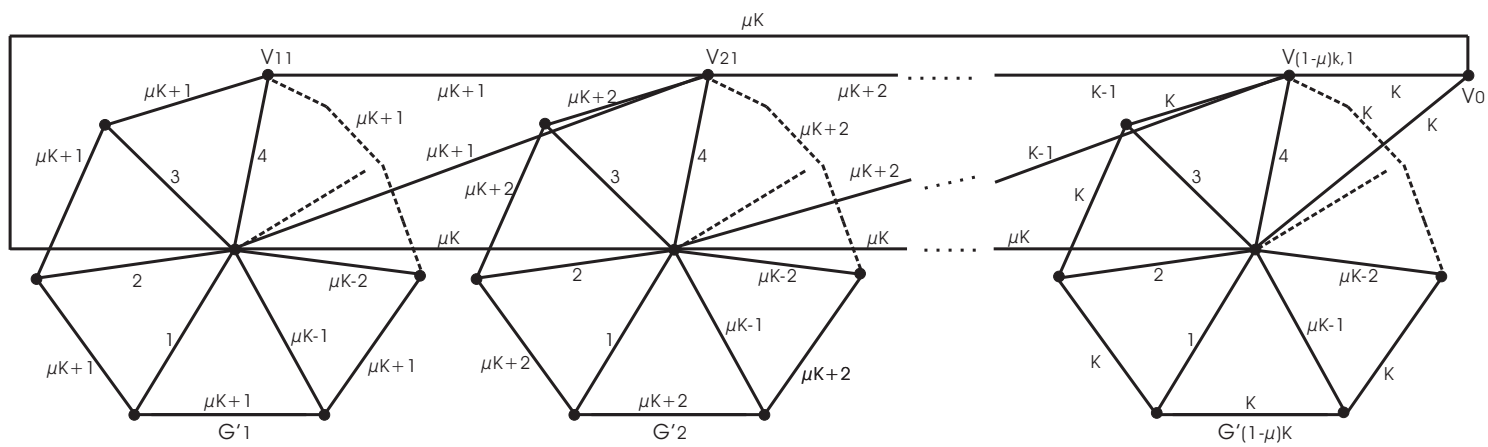

Fig. 1. Instance $G_{1}$.

Now, the all-zeros bit string with fitness vector $(n, 0)$ is included in the population. It is easy to see that $n \geq 2$, otherwise $G^{\prime}$ is trivial. According to Corollary 2, there is a label such that when it is added to the all-zeros bit string the number of connected components will be reduced to not more than $n\left(1-\frac{1}{2 \mathrm{OPT}\left(G^{\prime}\right)}\right)$. The probability of choosing this label is $\frac{1}{k}\left(1-\frac{1}{k}\right)^{k-1}=\Omega\left(\frac{1}{k}\right)$. Since the population size is $O(k)$, the probability that GSEMO selects the all-zeros bit string from $P$ is $\Omega\left(\frac{1}{k}\right)$. So a solution $X^{1}$ with fitness vector $\left(c_{1}, 1\right)$, where $c_{1} \leq\left\lfloor n\left(1-\frac{1}{2 \mathrm{OPT}\left(G^{\prime}\right)}\right)\right\rfloor \leq n\left(1-\frac{1}{2 \mathrm{OPT}\left(G^{\prime}\right)}\right)$ can be included in the population in expected runtime $O\left(k^{2}\right)$.

If $c_{1} \geq 2$, then there is still a label such that when it is added to $X^{1}$ the number of connected components will be reduced to not more than $n\left(1-\frac{1}{2 \mathrm{OPT}\left(G^{\prime}\right)}\right)^{2}$. So a solution $X^{2}$ with fitness vector $\left(c_{2}, 2\right)$, where $c_{2} \leq n\left(1-\frac{1}{2 \mathrm{OPT}\left(G^{\prime}\right)}\right)^{2}$ can be included in the population in expected runtime $O\left(k^{2}\right)$ after $X^{1}$ being included in the population.

Similarly, suppose that solution $X^{h-1}$ with fitness vector $\left(c_{h-1}, h-1\right)$, where $c_{h-1} \leq n\left(1-\frac{1}{2 \mathrm{OPT}\left(G^{\prime}\right)}\right)^{h-1}$, has now been included in the population. If $c_{h-1} \geq 2$, then a solution $X^{h}$ with fitness vector $\left(c_{h}, h\right)$, where $c_{h} \leq n\left(1-\frac{1}{2 \mathrm{OPT}\left(G^{\prime}\right)}\right)^{h}$, will be included in the population in expected runtime $O\left(k^{2}\right)$ after $X^{h-1}$ being included in the population.

Note that when $h=2 \cdot \operatorname{OPT}\left(G^{\prime}\right) \cdot \ln n, n\left(1-\frac{1}{2 \mathrm{OPT}\left(G^{\prime}\right)}\right)^{h} \leq$ 1. So, a connected spanning subgraph with at most 2 . $\operatorname{OPT}\left(G^{\prime}\right) \cdot \ln n$ labels will be finally included in the population in expected runtime $O\left(h k^{2}\right)=O\left(2 \cdot \mathrm{OPT}\left(G^{\prime}\right) \cdot k^{2} \ln n\right)=$ $O\left(k^{3} \ln n\right)$ after the all-zeros bit string being included in the population.

TABLE I

APPROXIMATION PERFORMANCES OF THE $(1+1)$ EA AND GSEMO. ' $r$ ' AND '- ' REFER TO THE APPROXIMATION RATIO AND UNKNOWN, RESPECTIVELY.

\begin{tabular}{l||c|c||c|c}
\hline \multicolumn{1}{l||}{} & \multicolumn{2}{c}{ The (1+1) EA } & GSEMO \\
\hline & $r$ & Runtime & $r$ & Runtime \\
\hline \hline MLST $_{b}$ & $\frac{b+1}{2}$ & $O\left(n k+k^{4}\right)$ & $\frac{b+1}{2}$ & $O\left(n k^{2}+k^{5}\right)$ \\
\hline MLST & - & - & $2 \ln n$ & $O\left(k^{2} \ln k+k^{3} \ln n\right)$ \\
\hline
\end{tabular}

Table I summarizes the approximation performances of the $(1+1)$ EA and GSEMO for the minimum label spanning tree problem. For the $\mathrm{MLST}_{b}$ problem, the $(1+1)$ EA and GSEMO can efficiently achieve a $\frac{b+1}{2}$-approximation ratio. However, the order of the upper bound on the expected runtime of
GSEMO is higher than that of the $(1+1)$ EA. This is because that GSEMO has to select a promising solution to mutate from a population of size $O(k)$. For the MLST problem, GSEMO efficiently achieves a $(2 \ln n)$-approximation ratio, but the approximation performance of the (1+1) EA is unknown.

\section{Performances of the $(1+1)$ EA AND GSEMO ON FOUR INSTANCES}

In this section, we first present an instance where GSEMO outperforms the (1+1) EA, then we show that the $(1+1)$ EA and GSEMO outperform local search algorithms on three instances of the MLST problem.

\section{A. An instance where GSEMO outperforms the $(1+1)$ EA}

In this subsection, we construct an instance $G_{1}=$ $\left\{V_{1}, E_{1}, L_{1}\right\}$ on which GSEMO is superior to the $(1+1)$ EA, where $L_{1}=\{1, \ldots, k\}$.

Given $\mu\left(0<\mu<\frac{1}{2}\right)$, we construct instance $G_{1}$ as follows. For simplicity, we assume that $\mu k$ is an integer, thus $(1-\mu) k$ is also an integer. First, we construct $(1-\mu) k$ subgraphs $G_{1}^{\prime}$, $\ldots, G_{(1-\mu) k}^{\prime} . G_{i}^{\prime}(1 \leq i \leq(1-\mu) k)$ contains a $(\mu k-1)$-sided regular polygon whose edges are all labeled by the same label $\mu k+i$ and an inner node in the center of this regular polygon. Since the number of sides of a regular polygon is at least $3, \mu k-1 \geq 3$, i.e., $\mu k \geq 4$. From the inner node of $G_{i}^{\prime}$, $(\mu k-1)$ edges labeled by from 1 to $\mu k-1$ connect to the $\mu k-1$ outer nodes $v_{i 1}, v_{i 2}, \ldots, v_{i, \mu k-1}$. Then three edges are connected from $G_{i}^{\prime}(1 \leq i \leq(1-\mu) k-1)$ to $G_{i+1}^{\prime}$ : the first one labeled by $\mu k+i$ is from the inner node of $G_{i}^{\prime}$ to outer node $v_{i+1,1}$ of $G_{i+1}^{\prime}$, the second one labeled by $\mu k+i$ is from outer node $v_{i 1}$ of $G_{i}^{\prime}$ to outer node $v_{i+1,1}$ of $G_{i+1}^{\prime}$, and the third one labeled by $\mu k$ is from the inner node of $G_{i}^{\prime}$ to the inner node of $G_{i+1}^{\prime}$. Finally, $v_{0}$ is connected to the inner node and $v_{(1-\mu) k, 1}$ of $G_{(1-\mu) k}^{\prime}$ with two edges labeled by $k$, and $v_{0}$ is also connected to the inner node of $G_{1}^{\prime}$ with an edge labeled by $\mu k$. Figure 1 shows instance $G_{1}$.

When $0<\mu<1 / 2, X_{1}^{*}=(\overbrace{1, \ldots, 1}^{\mu k}, \overbrace{0, \ldots, 0}^{(1-\mu) k})$ is the global optimum of $G_{1} . X_{1}^{l}=(\overbrace{0, \ldots, 0}^{\mu k}, \overbrace{1, \ldots, 1}^{(1-\mu) k})$ is a local optimum for LS2N. Since $X_{1}^{*}$ is the only one feasible solution that contains fewer labels than $X_{1}^{l}$. However, $\left|X_{1}^{l}-X_{1}^{*}\right|=(1-\mu) k$ and 
$\left|X_{1}^{*}-X_{1}^{l}\right|=\mu k$, which implies that $X_{1}^{*} \notin 2-S W I T C H\left(X_{1}^{l}\right)$ as $\mu k \geq 4$ and $(1-\mu) k>\mu k$.

For instance $G_{1}$, the expected runtime for the (1+1) EA to jump out of $X_{1}^{l}$ is exponential.

Theorem 4: For instance $G_{1}$, starting with $X_{1}^{l}$, the expected runtime for the $(1+1)$ EA to find the global optimum is $\Omega\left(k^{\mu k}\right)$.

Proof: For instance $G_{1}$, when the current solution is $X_{1}^{l}$, the $(1+1)$ EA only accepts the event that adds all $\mu k$ labels from $\{1, \ldots, \mu k\}$ and simultaneously removes more than $\mu k$ labels from $\{\mu k+1, \ldots, k\}$. So, the probability of escaping from the local optimum is

$$
\begin{aligned}
& \sum_{i=1}^{k-2 \mu k}\left(\begin{array}{c}
k-\mu k \\
\mu k+i
\end{array}\right)\left(\frac{1}{k}\right)^{2 \mu k+i}\left(1-\frac{1}{k}\right)^{k-2 \mu k-i} \\
& =\left(\frac{1}{k}\right)^{\mu k} \sum_{i=1}^{k-2 \mu k}\left(\begin{array}{l}
k-\mu k \\
\mu k+i
\end{array}\right)\left(\frac{1}{k}\right)^{\mu k+i}\left(1-\frac{1}{k}\right)^{k-2 \mu k-i} \\
& <\left(\frac{1}{k}\right)^{\mu k} .
\end{aligned}
$$

This is because $\sum_{i=1}^{k-2 \mu k}\left(\begin{array}{c}k-\mu k \\ \mu k+\end{array}\right)\left(\frac{1}{k}\right)^{\mu k+i}\left(1-\frac{1}{k}\right)^{k-2 \mu k-i}$

$$
\begin{aligned}
& <\sum_{i=1}^{k-2 \mu k}\left(\begin{array}{c}
k-\mu k \\
\mu k+i
\end{array}\right)\left(\frac{1}{k}\right)^{\mu k+i}\left(1-\frac{1}{k}\right)^{k-2 \mu k-i} \\
& +\sum_{i=-\mu k}^{0}\left(\begin{array}{c}
k-\mu k \\
\mu k+i
\end{array}\right)\left(\frac{1}{k}\right)^{\mu k+i}\left(1-\frac{1}{k}\right)^{k-2 \mu k-i} \\
& =\sum_{i=0}^{k-\mu k}\left(\begin{array}{c}
k-\mu k \\
i
\end{array}\right)\left(\frac{1}{k}\right)^{i}\left(1-\frac{1}{k}\right)^{k-\mu k-i}=1 .
\end{aligned}
$$

Thus, starting with $X_{1}^{l}$, the expected runtime for the $(1+1)$ EA to find the global optimum of $G_{1}$ is $\Omega\left(k^{\mu k}\right)$.

While the $(1+1)$ EA needs an expected exponential runtime to jump out of $X_{1}^{l}$, GSEMO can efficiently find the global optimum for instance $G_{1}$.

Theorem 5: For instance $G_{1}$, GSEMO finds the global optimum in expected runtime $O\left(k^{2} \ln k\right)$.

Proof: We first determine the expected runtime that GSEMO starting with any initial solution finds the all-zeros solution. Then we determine the expected runtime that starting with the all-zeros solution GSEMO produces the whole Pareto front.

Adding a label from $L_{1}^{\prime}=\{1, \ldots, \mu k\}$ to the all-zeros bit string can reduce the number of connected components by $(1-\mu) k$, while adding a label from $L_{1}^{\prime \prime}=\{\mu k+1, \ldots, k\}$ can reduce the number of connected components by $\mu k$. Note that when $0<\mu<1 / 2,(1-\mu) k$ is larger than $\mu k$. Hence, the Pareto front contains $\mu k+1$ Pareto optimal solutions with fitness vectors $(n, 0),(n-(1-\mu) k, 1), \ldots,(n-(1-\mu) j k, j)$, $\ldots,(1, \mu k)$, respectively. It is clear that the population size is $O(k)$.

It has been proved in Theorem 3 that the expected runtime for GSEMO starting with any initial solution to include the all-zeros bit string in the population is $O\left(k^{2} \ln k\right)$.

Now we calculate the expected runtime to produce the whole Pareto front after the all-zeros bit string being found. The worst case is from the all-zeros bit string to produce the whole Pareto front. Suppose that now in the population, there is a Pareto optimal solution $X$ with fitness vector $(n-(1-\mu) j k, j)$, which has the maximum number of labels. Another Pareto optimal solution with fitness vector $(n-(1-\mu)(j+1) k, j+1)$ can be produced by adding a label from $L_{1}^{\prime}$ which is not in $X$. The probability of adding this label is $\left(\begin{array}{c}\mu k-j \\ 1\end{array}\right) \frac{1}{k}\left(1-\frac{1}{k}\right)^{k-1} \geq \frac{\mu k-j}{e k}$. This implies that the expected runtime is $O\left(\frac{e k^{2}}{\mu k-j}\right)$, as the expected runtime that GSEMO selects $X$ from $P$ is $O(k)$. So, considering the worst case of starting with the all-zeros bit string, the expected runtime for GSEMO to produce the whole Pareto front is $\sum_{j=0}^{\mu k-1} \frac{e k^{2}}{\mu k-j}=O\left(k^{2} \ln k\right)$.

\section{$B$. An instance where the $(1+1)$ EA and GSEMO outperform} ERA

ERA is a local search algorithm. It takes an arbitrary spanning tree as input, then considers each non-tree edge and tests whether the number of used labels can be reduced by adding this non-tree edge and deleting a tree edge on the induced cycle.

In this subsection, we show that the $(1+1)$ EA and GSEMO outperform ERA on an instance proposed by Krumke and Wirth [15], which is denoted by $G_{2}$ in this paper.

This instance can be constructed in two steps. First, a star shaped graph is constructed by selecting one node out of $n$ nodes and connecting it to the other $n-1$ nodes with $n-1$ edges, which are labeled by $n-1$ distinct labels: $1,2, \ldots$, $n-1$. Second, a complete graph is constructed by adding edges with the same label $k$ to the star shaped graph. Thus, we get a complete graph $G_{2}=\left(V_{2}, E_{2}, L_{2}\right)$, where $\left|V_{2}\right|=n$, $\left|E_{2}\right|=n(n-1) / 2$, and $L_{2}=\{1,2, \ldots, k\}$ is the set of labels. It is clear that $\left|L_{2}\right|=k$ and $k=n$. Figure 2 shows an example with $n=5$, where the dashed edges construct a spanning tree with the minimum number of labels.

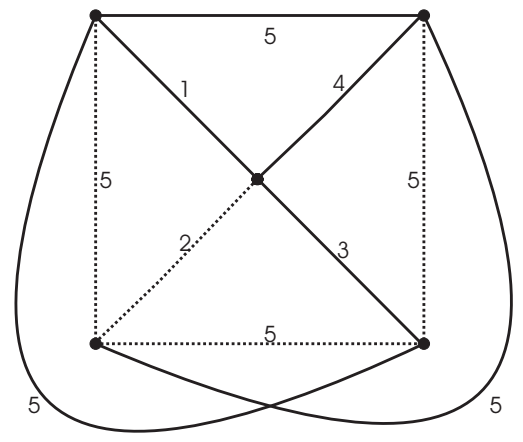

Fig. 2. An example of instance $G_{2}$ with $n=5$.

For instance $G_{2}$, the global optimum $X_{2}^{*}=\left(x_{21}^{*}, \ldots, x_{2 k}^{*}\right)$ contains two labels: one comes from $\{1, \ldots, k-1\}$ and the other is label $k$, i.e., $\left|X_{2}^{*}\right|=2, \sum_{i=1}^{k-1} x_{2 i}^{*}=1$, and $x_{2 k}^{*}=1$.

Krumke and Wirth used instance $G_{2}$ to demonstrate that ERA might perform as badly as possible. In fact, $X_{2}^{l}=$

$(\overbrace{1, \ldots, 1}^{k-1}, 0)$ is a local optimum for ERA, since starting with $X_{2}^{l}$ the number of labels used in $H\left(X_{2}^{l}\right)$ cannot be reduced by adding any non-tree edge and deleting a tree edge on the induced cycle. The local optimum uses $k-1$ labels, while the global optimum uses only 2 labels. However, the $(1+1)$ EA can efficiently solve instance $G_{2}$.

Theorem 6: For instance $G_{2}$, the $(1+1)$ EA starting with any initial solution finds a global optimum in expected runtime $O(k \ln k)$.

Proof: For simplicity, let $L_{2}^{\prime}$ denote the label set $\{1, \ldots, k-1\}$. 
Let $A=\left\{X \mid c\left(H(X)=1, x_{k}=1,2 \leq|X| \leq k-1\right\}\right.$, i.e., a solution $X \in A$ contains label $k$ and at least one but at most $k-2$ labels from $L_{2}^{\prime}$. Obviously, any solution $X \in A$ is feasible.

To find a global optimum, a solution $X \in A$ should be found first. Once a solution $X \in A$ has been found, the global optimum can be found by removing all $|X|-2$ redundant labels from $L_{2}^{\prime}$. Once such a label is removed from $X$, it cannot be added anymore. According to the Coupon Collector's theorem [39], all redundant labels contained in $X$ will be removed in expected runtime $O(k \ln k)$.

In order to analyze the expected runtime to find a solution $X \in A$, we further partition all solutions that do not belong to $A$ into five disjoint subsets $B_{1}, B_{2}, B_{3}, B_{4}, B_{5}$ :

$B_{1}=\left\{X|c(H(X))=1| X \mid,=k\right.$, and $\left.x_{k}=1\right\}$;

$B_{2}=\left\{X|c(H(X))=1| X \mid,=k-1\right.$, and $\left.x_{k}=0\right\}$;

$B_{3}=\left\{X|c(H(X))>1,1 \leq| X \mid \leq k-2\right.$, and $\left.x_{k}=0\right\}$;

$B_{4}=\left\{X|c(H(X))>1| X \mid,=1\right.$, and $\left.x_{k}=1\right\}$;

$B_{5}=\{X|c(H(X))>1| X \mid,=0\}$.

If $X \in B_{1}$, then $X$ will be transformed into $A$ by removing one label from $L_{2}^{\prime}$. The probability of this event is $\left(\begin{array}{c}k-1 \\ 1\end{array}\right) \frac{1}{k}(1-$ $\left.\frac{1}{k}\right)^{k-1}=\Omega(1)$, which implies that the expected runtime is $O(1)$.

If $X \in B_{2}$, then $X$ will be transformed into $A$ by adding label $k$ and simultaneously removing one label from $L_{2}^{\prime}$. The probability of this event is $\left(\begin{array}{c}k-1 \\ 1\end{array}\right)\left(\frac{1}{k}\right)^{2}\left(1-\frac{1}{k}\right)^{k-2}=\Omega\left(\frac{1}{k}\right)$, which implies that the expected runtime is $O(k)$.

If $X \in B_{3}\left(B_{4}\right)$, then $X$ will be transformed into $A$ by adding label $k$ (one label from $L_{2}^{\prime}$ ). The probability of this event is $\frac{1}{k}\left(1-\frac{1}{k}\right)^{k-1}=\Omega\left(\frac{1}{k}\right)\left(\left(\begin{array}{c}k-1 \\ 1\end{array}\right) \frac{1}{k}\left(1-\frac{1}{k}\right)^{k-1}=\Omega(1)\right)$, which implies that the expected runtime is $O(k)$.

If $X \in B_{5}$, then $X$ will be transformed into $A$ by simultaneously adding label $k$ and a label from $L_{2}^{\prime}$. The probability is $\left(\begin{array}{c}k-1 \\ 1\end{array}\right)\left(\frac{1}{k}\right)^{2}\left(1-\frac{1}{k}\right)^{k-2}=\Omega\left(\frac{1}{k}\right)$, which implies that the expected runtime is $O(k)$.

So, any solution will be transformed into $A$ in expected runtime $O(k)$.

Combining the expected runtime to remove all redundant labels contained in a solution belonging to $A$, the expected runtime for the $(1+1)$ EA starting with any initial solution to find a global optimum is $O(k \ln k)$.

As shown in the following theorem, GSEMO can also efficiently solve instance $G_{2}$.

Theorem 7: For instance $G_{2}$, GSEMO starting with any initial solution finds a global optimum in expected runtime $O\left(k^{2} \ln k\right)$.

Proof: Let $L_{2}^{\prime}$ denote the label set $\{1, \ldots, k-1\}$. The optimization process consists of two independent phases: the first phase lasts until a solution with fitness vector $(1, *)$, i.e., a feasible solution, is included in the population, and the second phase ends when a global optimum is found.

We now analyze the expected runtime of the first phase. Let $X$ be the solution with fitness vector $(c(H(X)),|X|)$, where $c(H(X))$ is the minimum among all solutions in the population. If $c(H(X))>1$, then there are three cases. The first one is that $X$ contains no label, the second is that $X$ contains label $k$ but no label from $L_{2}^{\prime}$, and the third is that $X$ contains at least one but at most $k-2$ labels from $L_{2}^{\prime}$ and no label $k$.

For all three cases, a solution with fitness vector $(1, *)$ will be included in expected runtime $O\left(k^{2}\right)$, since the probability of selecting $X$ from $P$ is $\Omega\left(\frac{1}{k}\right)$, and the probability of transforming $X$ into a solution with fitness vector $(1, *)$ is $\Omega\left(\frac{1}{k}\right)$.

Once a solution with fitness vector $(1, *)$ is included in the population, we show that a global optimum will be found in expected runtime $O\left(k^{2} \ln k\right)$. To this end, we partition the second phase into two subphases: the first subphase lasts until a solution belonging to $A=\left\{X\left|x_{k}=1,2 \leq\right| X \mid \leq k-1\right\}$ is found, i.e, such a solution contains label $k$ and at least one but at most $k-2$ labels from $L_{2}^{\prime}$, the second subphase ends when a global optimum is found.

If a solution $X$ with fitness vector $(1, *)$ and $X \notin A$, then there are two cases needed to be considered: the first is that $X$ contains label $k$ and all labels from $L_{2}^{\prime}$, and the other is that $X$ contains all labels from $L_{2}^{\prime}$ excluding label $k$.

For the first case, removing anyone of labels from $L_{2}^{\prime}$ will transform $X$ into $A$. The probability of this event is $\left(\begin{array}{c}k-1 \\ 1\end{array}\right) \frac{1}{k}\left(1-\frac{1}{k}\right)^{k-1}=\Omega(1)$, which implies that the expected runtime is $O(1)$. For the second case, removing one label from $L_{2}^{\prime}$ and simultaneously adding label $k$ will transform $X$ into $A$. The probability of this event is $\left(\begin{array}{c}k-1 \\ 1\end{array}\right)\left(\frac{1}{k}\right)^{2}\left(1-\frac{1}{k}\right)^{k-2}=\Omega\left(\frac{1}{k}\right)$, which implies that the expected runtime is $O(k)$. Noting that the probability of selecting $X$ from the population is $\Omega\left(\frac{1}{k}\right)$, a solution belonging to $A$ will be included in the population in expected runtime $O\left(k^{2}\right)$ after a solution with fitness vector $(1, *)$ being included.

Now at least one solution belonging to $A$ is included in the population. Let $X$ be the solution which has the minimum number of labels among all solutions in the population belonging to $A$. If $X$ is not the global optimum of $G_{2}$, then the global optimum will be found by removing all $|X|-2$ redundant labels from $L_{2}^{\prime}$. Once such a label is removed from $X$, it cannot be added anymore. According to the Coupon Collector's theorem [39], all redundant labels will be removed in expected runtime $O(k \ln k)$, and the probability of selecting $X$ from $P$ is $\Omega\left(\frac{1}{k}\right)$, so a global optimum will be found in expected runtime $O\left(k^{2} \ln k\right)$.

Altogether, GSEMO starting with any initial solution finds a global optimum in expected runtime $O\left(k^{2} \ln k\right)$.

\section{An instance where the $(1+1)$ EA and GSEMO outperform $L S 2 N$}

Brüggemann, Monnot, and Woeginger proposed an instance, denoted by $G_{3}$ in this paper, to show that there exists a local optimum with respect to LS2N [36].

As shown in Figure 3, this instance is a graph $G_{3}=$ $\left(V_{3}, E_{3}, L_{3}\right)$, where $V_{3}=\left(v_{0}, x_{0}, x_{1}, \ldots, x_{k-4}, y_{0}, y_{1}\right.$, $\left.\ldots, y_{k-4}\right), L_{3}=(1,2, \ldots, k),\left|V_{3}\right|=2 k-5,\left|E_{3}\right|=4 k-12$, and $\left|L_{3}\right|=k$. Figure 4 shows the minimum label spanning tree. $1)$. 


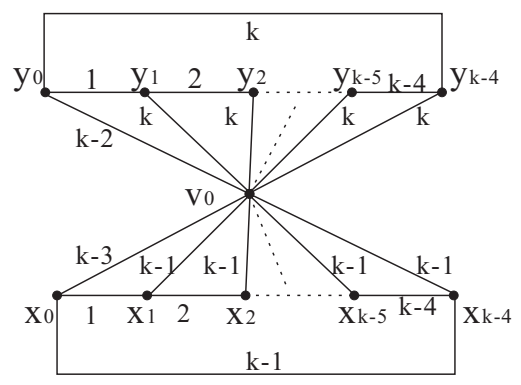

Fig. 3. Instance $G_{3}$.

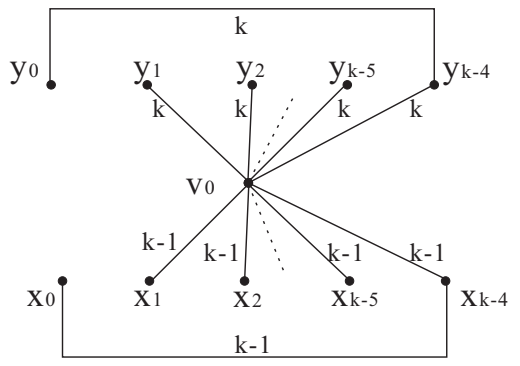

Fig. 4. The MLST of instance $G_{3}$.

LS2N might be trapped in the local optimum which contains labels $1,2, \ldots, k-2$. In fact, to jump out of this local optimum, at least three labels from $\{1,2, \ldots, k-2\}$ should be removed and simultaneously two labels $k-1$ and $k$ should be added, but the resulting solution is not in the 2 -switch neighborhood of the local optimum. Hence, L2SN may not find the global optimum of $G_{3}$. However, the (1+1) EA is efficient for instance $G_{3}$.

Theorem 8: For instance $G_{3}$, the $(1+1)$ EA starting with any initial solution finds the global optimum in expected runtime $O\left(k^{2}\right)$.

Proof: Let $L_{3}^{\prime}$ denote the label set $\{1, \ldots, k-2\}$, and let $C=\left\{X\left|c(H(X))=1, x_{k-1}=1, x_{k}=1,2 \leq\right| X \mid \leq k-1\right\}$, i.e, a solution $X \in C$ contains labels $k-1$ and $k$ and at most $k-3$ labels from $L_{3}^{\prime}$.

Note that the global optimum contains only two labels $k-1$ and $k$. The optimization process consists of two independent phases: the first phase lasts until a solution $X \in C$ is constructed from an arbitrary solution, and the second phase ends when all $|X|-2$ redundant labels from $L_{3}^{\prime}$ are removed.

For analyzing the expected runtime of finding a solution $X \in C$, we partition all solutions that do not belong to $C$ into seven disjoint subsets $D_{1}, D_{2}, D_{3}, D_{4}, D_{5}, D_{6}, D_{7}$ :

$D_{1}=\left\{X \mid c\left(H(X)=1, x_{k-1}=0, x_{k}=0,|X|=k-2\right\}\right.$;

$D_{2}=\left\{X \mid c\left(H(X)=1, x_{k-1}=0, x_{k}=1,|X|=k-2\right.\right.$ or $|X|=k-1\}$;

$D_{3}=\left\{X \mid c\left(H(X)=1, x_{k-1}=1, x_{k}=0,|X|=k-2\right.\right.$ or

$|X|=k-1\}$;

$D_{4}=\left\{X \mid c\left(H(X)=1, x_{k-1}=1, x_{k}=1,|X|=k\right\}\right.$;

$D_{5}=\left\{X \mid c\left(H(X)>1, x_{k-1}=0, x_{k}=0\right\}\right.$;

$D_{6}=\left\{X \mid c\left(H(X)>1, x_{k-1}=0, x_{k}=1\right\}\right.$;
$D_{7}=\left\{X \mid c\left(H(X)>1, x_{k-1}=1, x_{k}=0\right\}\right.$.

If $X \in D_{1}$, then $X$ will be transformed into $C$ by adding labels $k-1, k$, and simultaneously removing three labels from $L_{3}^{\prime}$. The probability of this event is $\left(\begin{array}{c}k-2 \\ 3\end{array}\right)\left(\frac{1}{k}\right)^{5}\left(1-\frac{1}{k}\right)^{k-5}=$ $\Omega\left(\frac{1}{k^{2}}\right)$, which implies that the expected runtime is $O\left(k^{2}\right)$.

If $X \in D_{2}\left(D_{3}\right)$, then $X$ will be transformed into $C$ by adding label $k-1(k)$ and simultaneously removing two labels from $L_{3}^{\prime}$. The probability of this event is $\left(\begin{array}{c}k-3 \\ 2\end{array}\right)\left(\frac{1}{k}\right)^{3}(1-$ $\left.\frac{1}{k}\right)^{k-3}=\Omega\left(\frac{1}{k}\right)$, which implies that the expected runtime is $O(k)$.

If $X \in D_{4}$, then $X$ will be transformed into $C$ by removing a label from $L_{3}^{\prime}$. The probability of this event is $\left(\begin{array}{c}k-2 \\ 1\end{array}\right) \frac{1}{k}(1-$ $\left.\frac{1}{k}\right)^{k-1}=\Omega(1)$, which implies that the expected runtime is $O(1)$.

If $X \in D_{5}$, then $X$ will be transformed into $C$ by simultaneously adding labels $k-1$ and $k$. The probability of this event is $\left(\frac{1}{k}\right)^{2}\left(1-\frac{1}{k}\right)^{k-2}=\Omega\left(\frac{1}{k^{2}}\right)$, which implies that the expected runtime is $O\left(k^{2}\right)$.

If $X \in D_{6}\left(D_{7}\right)$, then $X$ will be transformed into $C$ by adding label $k-1(k)$. The probability of this event is $\frac{1}{k}(1-$ $\left.\frac{1}{k}\right)^{k-1}=\Omega\left(\frac{1}{k}\right)$, which implies that the expected runtime is $O(k)$.

So, a solution belonging to $C$ will be found in expected runtime $O\left(k^{2}\right)$.

In the second phase, removing each label contained in a solution belonging to $C$ which is from $L_{3}^{\prime}$ will reduce the fitness value, and once it is removed it cannot be added anymore. According to the Coupon Collectors theorem [39], the second phase ends in expected runtime $O(k \ln k)$.

Altogether, the expected runtime for the $(1+1)$ EA starting with any initial solution to find the global optimum is $O\left(k^{2}\right)$.

GSEMO is also efficient for instance $G_{3}$.

Theorem 9: For instance $G_{3}$, the expected runtime for GSEMO starting with any initial solution to find the global optimum is $O\left(k^{2} \ln k\right)$.

Proof: We first analyze the runtime that the all-zeros solution is found by GSEMO starting with an arbitrary solution, then analyze the runtime that the global optimum is found by GSEMO once the all-zeros solution is included in the population.

It has been proved in Theorem 3 that the expected runtime for GSEMO starting with any initial solution to find the allzeros bit string is $O\left(k^{2} \ln k\right)$.

Once the all-zeros bit string is included in the population, the Pareto optimal solution $X^{1}$ with fitness vector $(k-2,1)$ will be found by adding label $k-1$ or $k$ to the all-zeros bit string. Since the probability that GSEMO selects the all-zeros bit string from $P$ is $\Omega\left(\frac{1}{k}\right)$, and the probability of only flipping a bit corresponding to anyone of such labels is $\frac{2}{k}\left(1-\frac{1}{k}\right)^{k-1}=$ $\Omega\left(\frac{1}{k}\right)$. So, $X^{1}$ will be included in the population in expected runtime $O\left(k^{2}\right)$ after the all-zeros bit string being included. Then the global optimum $X_{3}^{*}$ with fitness vector $(1,2)$ will be found by adding the remaining label from $\{k-1, k\}$ to solution $X^{1}$, and the expected runtime to produce solution $X_{3}^{*}$ from solution $X^{1}$ is also $O\left(k^{2}\right)$.

Therefore, the expected runtime for GSEMO starting with any initial solution to find the global optimum is $O\left(k^{2} \ln k\right)$. 


\section{An instance where the $(1+1)$ EA and GSEMO outperform the modified MVCA}

In this subsection, we show that the (1+1) EA and GSEMO outperform the modified MVCA on an instance proposed by Xiong, Golden, and Wasil [17], which is denoted by $G_{4}$ in this paper.

Given the bound of the labels' frequency $b(b \geq 2)$, let $n=$ $b \cdot b !+1$. We construct $G_{4}=\left(V_{4}, E_{4}, L_{4}\right)$ as follows, where $V_{4}=\{1,2, \ldots, n\},\left|V_{4}\right|=n$, and $L_{4}=I_{b} \cup I_{b-1} \cup \ldots \cup I_{2} \cup I^{\prime}$.

We construct $b$ ! groups from $V_{4}$, each containing $b+1$ nodes: $V_{1}^{\prime}=\{1,2, \ldots, b+1\}$

$V_{2}^{\prime}=\{b+1, b+2, \ldots, 2 b+1\}$,

.

$V_{j}^{\prime}=\{(j-1) b+1,(j-1) b+2, \ldots, j b+1\}$,

...

$V_{b !}^{\prime}=\{(b !-1) b+1,(b !-1) b+2, \ldots, b ! b+1\}$.

In $V_{j}^{\prime}(j=1,2, \ldots, b !)$, all pairs of consecutive nodes $((j-$ 1) $b+1,(j-1) b+2), \ldots,(j b, j b+1)$ are connected by $b$ edges, and all these $b$ edges are labeled by one label. Thus, $b$ ! labels are needed, which constitute the label set $I^{\prime}$.

In each $V_{j}^{\prime}$, node $(j-1) b+1$ is connected to nodes $(j-$ 1) $b+3, \ldots, j b+1$. The label subset $I_{h}(h=b, b-1, \ldots, 2)$ is obtained as follows. We choose edge $((j-1) b+1,(j-$ 1) $b+1+h)$ in each $V_{j}^{\prime}$, so there are $b$ ! such edges. We label the first $h$ edges with one label, and the next $h$ edges with a second label, and so on. So, $\frac{b !}{h}$ labels are needed, and they construct $I_{h}$.

Hence, $\left|I_{h}\right|=\frac{b !}{h}(h=b, b-1, \ldots, 2),\left|I^{\prime}\right|=b !$, and the total number of labels $k=\sum_{j=2}^{b} \frac{b !}{j}+b$ !. The edges with $b$ ! labels from $I^{\prime}$ construct the minimum label spanning tree, so in this instance the global optimum is $X_{4}^{*}=(\overbrace{0, \ldots, 0}^{\sum_{j=2}^{b}=\overbrace{1, \ldots, 1}^{\frac{b !}{j}}} \overbrace{1, \ldots}^{b !})$.

Figure 5 shows an example with $b=4$, where the dashed edges construct the spanning tree with the minimum number of labels.

Xiong, Golden, and Wasil used this instance to show that the modified MVCA may obtain a solution using all labels from $I_{b} \cup I_{b-1} \cup \ldots \cup I_{2} \cup I^{\prime}$, which is $H_{b}$-approximation solution, where $H_{b}=\sum_{i=1}^{b} \frac{1}{i}$. However, the $(1+1)$ EA can efficiently find the global optimum of instance $G_{4}$.

Theorem 10: For instance $G_{4}$, the $(1+1)$ EA starting with any initial solution finds the global optimum in expected runtime $O(n k)$, where $n=b \cdot b !+1, k=\sum_{j=2}^{b} \frac{b !}{j}+b !$ and $b$ is the maximum frequency of the labels.

Proof: The optimization process consists of two independent phases. The first phase ends when the $(1+1)$ EA finds a feasible solution, and the second phase lasts until the $(1+1)$ EA removes all redundant labels from $\left\{1,2, \ldots, \sum_{j=2}^{b} \frac{b !}{j}\right\}$.

Let $X$ be the current solution. Note that a feasible solution contains all labels from $I^{\prime}$. If $X$ is not a feasible solution, then there must exist a bit $x_{h}$ from $\left\{x_{i} \mid \sum_{j=2}^{b} \frac{b !}{j}+1 \leq i \leq\right.$ $\sum_{j=2}^{b} \frac{b !}{j}+b$ ! $\}$ whose value is 0 . So, the $(1+1)$ EA can decrease the number of connected components by at least one with probability $\frac{1}{k}\left(1-\frac{1}{k}\right)^{k-1} \geq \frac{1}{e k}$. This is the probability of the event that bit $x_{h}$ is flipped from 0 to 1 and the other bits keep unchanged. Hence, the expected runtime to decrease the number of connected components from $n$ to 1 is $O(n k)$, i.e., a feasible solution will be found by the $(1+1)$ EA in expected runtime $O(n k)$.

Once a feasible solution $X$ is found, each bit from $\left\{x_{i} \mid \sum_{j=2}^{b} \frac{b !}{j}+1 \leq i \leq \sum_{j=2}^{b} \frac{b !}{j}+b !\right\}$ takes value 1 , and the flippings of them cannot be accepted by the $(1+1)$ EA, as such flippings will create a disconnected spanning subgraph. For each bit $x_{i}\left(1 \leq i \leq \sum_{j=2}^{b} \frac{b !}{j}\right)$, if $x_{i}=1$, then it can be flipped from 1 to 0 , since this will decrease the fitness value; otherwise, its flipping cannot be accepted by the $(1+1)$ EA, as this will increase the fitness value. So, when all bits have been selected at least once to flip, the global optimum will be found. According to the Coupon Collector's theorem [39], the expected runtime for this to happen is $O(k \ln k)$.

Hence, the expected runtime for the $(1+1)$ EA starting with any initial solution to find the global optimum is $O(n k+k \ln k)$ $=O(n k)$. Note that $n=b \cdot b !+1>k=b !\left(1+\frac{1}{2}+\ldots+\frac{1}{b}\right)$, and $k>\ln k$. So, $n>\ln k$, and $n k>k \ln k$.

GSEMO can solve instance $G_{4}$ in expected runtime polynomial in $k$.

Theorem 11: For instance $G_{4}$, GSEMO starting with any initial solution finds the global optimum in expected runtime $O\left(k^{3}\right)$.

Proof: The optimization process consists of two phases. The first phase lasts until GSEMO starting with any initial solution finds a solution with fitness vector $(1, *)$, and the second phase ends when GSEMO finds the global optimum.

Note that a connected spanning subgraph contains all labels from $I^{\prime}$. If $X$ is a solution such that $c(H(X))>1$, then at least one label from $I^{\prime}$ is not contained in it, and the number of connected components can be decreased by adding such a label.

We now analyze the expected runtime that GSEMO starting with any initial solution finds a solution with fitness vector $(1, *)$. If such a solution has not been included in the population, then there is a solution $X$ from $P$ such that $c(H(X))$ is the minimal, and adding some label $l$ from $I^{\prime}$ to $X$ will reduce the number of connected components. The probability that GSEMO chooses $X$ from $P$ is $\Omega\left(\frac{1}{k}\right)$, as the population size is $O(k)$, and the probability of flipping only the bit corresponding to label $l$ is $\frac{1}{k}\left(1-\frac{1}{k}\right)^{k-1}=\Omega\left(\frac{1}{k}\right)$, so a solution with a smaller number of connected components will be found in expected runtime $O\left(k^{2}\right)$. After all labels from $I^{\prime}$ being added, a connected spanning subgraph will be constructed. Thus a solution with fitness vector $(1, *)$ will included in expected runtime $O\left(b ! k^{2}\right)=O\left(k^{3}\right)$.

Now at least one solution with fitness vector $(1, *)$ is included in the population. Let $X$ be the solution which has the minimum number of labels among all solutions in the population with fitness vector $(1, *)$. If $X$ is not the global optimum of $G_{4}$, then GSEMO can finish the second phase by removing all redundant labels from $I_{b} \cup I_{b-1} \cup \ldots \cup I_{2}$. Since the probability of selecting $X$ form $P$ is $\Omega\left(\frac{1}{k}\right)$, and removing all redundant labels needs an expected runtime $O(k \ln k)$, the global optimum will be found in expected runtime $O\left(k^{2} \ln k\right)$.

Combining the expected runtime in two phases, we finish 

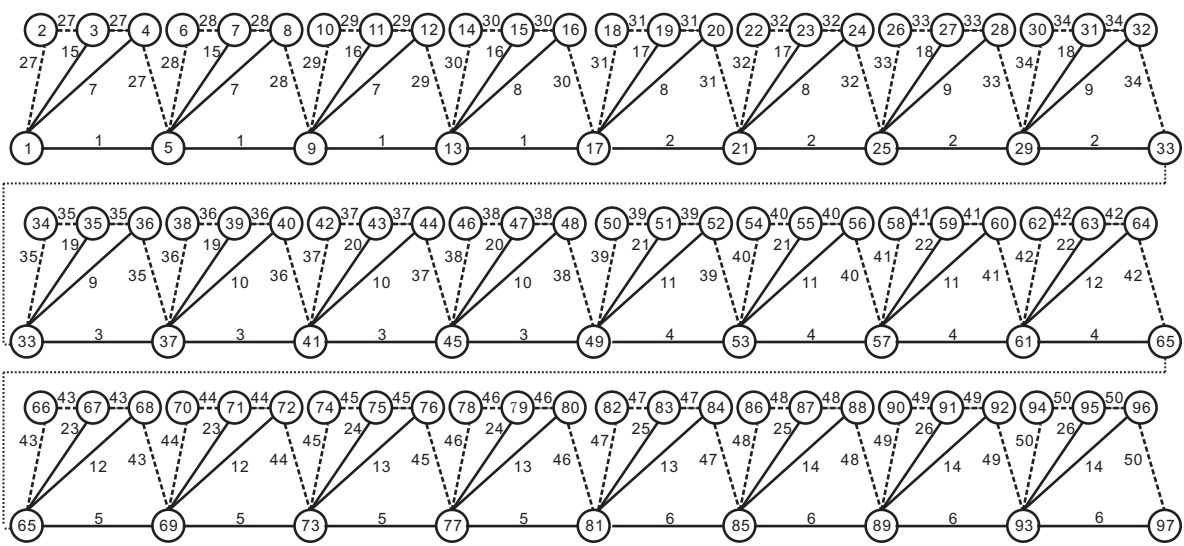

Fig. 5. Instance $G_{4}$ with $\mathrm{b}=4$.

the proof.

TABLE II

UPPER BOUNDS ON THE EXPECTED RUNTIME OF THE $(1+1)$ EA, GSEMO, ERA, LS2N, AND THE MODIFIED MVCA TO FIND THE GLOBAL OPTIMA ON FOUR INSTANCES. '- ' AND 'INF' MEAN UNKNOWN AND INFINITE, RESPECTIVELY.

\begin{tabular}{l||c|c|c|c}
\hline & Instance $G_{1}$ & Instance $G_{2}$ & Instance $G_{3}$ & Instance $G_{4}$ \\
\hline \hline The (1+1) EA & $\Omega\left(k^{\mu k}\right)$ & $O(k \ln k)$ & $O\left(k^{2}\right)$ & $O(n k)$ \\
\hline GSEMO & $O\left(k^{2} \ln k\right)$ & $O\left(k^{2} \ln k\right)$ & $O\left(k^{2} \ln k\right)$ & $O\left(k^{3}\right)$ \\
\hline ERA & - & $\operatorname{Inf}$ & - & - \\
\hline LS2N & - & - & Inf & - \\
\hline The modified MVCA & - & - & - & Inf \\
\hline
\end{tabular}

Note: $\Omega\left(k^{\mu k}\right)$ is the lower bound on the expected runtime that the $(1+1)$ EA starting with the given initial solution $X_{1}^{l}$ finds the global optimum of $G_{1}$.

Table II shows that GSEMO outperforms the $(1+1)$ EA on $G_{1}$. This is mainly because that GSEMO behaves greedily.

It also shows that ERA (LS2N, the modified MVCA) may be trapped in the local optimum of $G_{2}\left(G_{3}, G_{4}\right)$, thus it cannot efficiently solve $G_{2}\left(G_{3}, G_{4}\right)$. However, the $(1+1)$ EA and GSEMO can efficiently solve them.

This theoretically shows that EAs outperform local search algorithms on some instances. The reason is that EAs are global search algorithms as they use global mutation, while a local search algorithm searches the neighborhood of a current solution, thus it may be trapped in a local optimum.

Nevertheless, local search algorithms are not always worse than EAs.

For example, the modified MVCA can efficiently solve instance $G_{2}$. Recall that the modified MVCA begins with the all-zeros bit string, then chooses at each step a label such that when this label is chosen the decrease in the number of connected components is the largest. Therefore, the modified MVCA first tests all labels and chooses label $k$, as when it is chosen the decrease in the number of connected components is the largest. Next, the modified MVCA tests the remaining $k-1$ labels: $1,2, \ldots, k-1$, and randomly chooses one of them as the decrease in the number of connected components is the same when each of them is chosen. Hence, a global optimum of $G_{2}$ is found by the modified MVCA in runtime $2 k-1$ which is superior to that of the $(1+1)$ EA and GSEMO.

\section{CONCLUSION}

In this paper, we investigate the performances of the $(1+1)$ EA and GSEMO for the minimum label spanning tree problem. We reveal that the $(1+1)$ EA and GSEMO can guarantee to achieve some approximation ratios for the MLST problem. This gives a nice example to show that EAs can guarantee to achieve some certain approximation ratios for difficult NPhard problems. We also theoretically show that the $(1+1)$ EA and GSEMO defeat local search algorithms on some instances, and that GSEMO outperforms the $(1+1)$ EA on an instance.

On the $\mathrm{MLST}_{b}$ problem, the (1+1) EA and GSEMO achieve the same approximation ratio of $\frac{b+1}{2}$. To find a $\frac{b+1}{2}$ approximation solution for the $\mathrm{MLST}_{b}$ problem, the upper bound on the expected runtime of GSEMO is larger than that of the $(1+1)$ EA. However, we still have no idea how to obtain the lower bounds on their runtime for such an approximation ratio.

As for the approximation ratio of the $(1+1)$ EA on the MLST problem, we still know nothing about it. Apart from this, since the $(1+1)$ EA and GSEMO are randomized algorithms, it is natural to ask whether they can achieve better approximate ratios than those guaranteed by some other algorithms or not.

Usually, EAs in practice use a population of individuals, and in order to retain the diversity of individuals an appropriate population size is needed, which is different from the $(1+1)$ EA using only one individual. Thus, the performance analysis of population-based EAs is an important research topic, e.g., [19], [40], [41], [42].

How do population-based EAs such as $(\mu+\lambda)$ EAs perform on the MLST problem? Are they better than the $(1+1)$ EA? Crossover is usually an important operator for populationbased EAs. Several theoretical investigations have recently proven that crossover is essential or useful on some problems, e.g., [43], [44]. Therefore, it is interesting to investigate the performance of the population-based EA using not only mutation but also crossover on the MLST problem.

\section{REFERENCES}

[1] A. S. Tanenbaum, Computer Networks. Englewood Cliffs, NJ: Prentice Hall, 1989. 
[2] R.-S. Chang and S.-J. Leu, "The minimum labeling spanning trees," Inform. Process. Lett., vol. 63, no. 5, pp. 277-282, 1997.

[3] A. Chwatal, G. Raidl, and O. Dietzel, "Compressing fingerprint templates by solving an extended minimum label spanning tree problem," in Proc. 7th Metaheuristics International Conference (MIC'07), Montreal, Canada, Jun. 2007, pp. 105/1-3.

[4] A. Chwatal, G. Raidl, and K. Oberlechner, "Solving a $k$-node minimum label spanning arborescence problem to compress fingerprint Templates," J. Math. Mod. Algor., vol. 8, pp. 293-334, 2009.

[5] J. Holland, Adaptation in Natural and Artificial Systems. Ann Arbor, MI: University of Michigan Press, 1975.

[6] D. Goldberg. Genetic Algorithms in Search, Optimization, and Machine Learning. New York, NY: Addison-Wesley, 1989.

[7] F. Herrera, M. Lozano and J. Verdegay, "Tackling real-coded genetic algorithms: Operators and tools for behavioural analysis," Artif. Intell. Rev., vol. 12, no. 4, pp. 265-319, 1998.

[8] K. Gallagher and M. Sambridge, "Genetic algorithms: a powerful tool for large-scale non-linear optimization problems," Comput. Geosci., vol. 20, no. 7-8, pp. 1229-1236, 1994.

[9] Y. Xiong, B. Golden, and E. Wasil, "A one-parameter genetic algorithm for the minimum labeling spanning tree problem," IEEE Trans. Evol. Comput., vol. 9, no. 1, pp. 55-60, 2005.

[10] J. Nummela and B. Julstrom, "An effective genetic algorithm for the minimum-label spanning tree problem," in Proc. Genetic Evol. Comput. Conf. (GECCO'06), Seattle, Washington, Jul. 2006, pp. 553-558.

[11] S. Consoli and J. Moreno-Pérez, "Solving the minimum labelling spanning tree problem using hybrid local search," Electronic Notes in Discrete Mathematics, vol. 39, pp. 75-82, 2012.

[12] A. Chwatal and G. Raidl, "Solving the minimum label spanning tree problem by mathematical programming techniques," Vienna University of Technology, Institute of Computer Graphics and Algorithms, Tech. Rep. TR 186-1-10-03, Jun. 2010.

[13] R. Cerulli, A. Fink, M. Gentili, and S. Voß, "Metaheuristics comparison for the minimum labelling spanning tree problem," in The Next Wave on Computing, Optimization, and Decision Technologies, B. L. Golden, S. Raghavan, E. A. Wasil, eds. New York: Springer, 2005, pp. 93-106.

[14] S. Consoli, K. Darby-Dowman, N. Mladenović, and J. Moreno-Pérez, "Greedy randomized adaptive search and variable neighbourhood search for the minimum labelling spanning tree problem," Eur. J. Oper. Res., vol. 196, pp. 440-449, 2009.

[15] S. O. Krumke and H. Wirth, "On the minimum label spanning tree problem," Inform. Process. Lett., vol. 66, no. 2, pp. 81-85, 1998.

[16] Y. Wan, G. Chen, and Y. Xu, "A note on the minimum label spanning tree," Inform. Process. Lett., vol. 84, pp. 99-101, 2002.

[17] Y. Xiong, B. Golden, and E. Wasil, "Worst-case behavior of the MVCA heuristic for the minimum labeling spanning tree problem," Oper. Res. Lett., vol. 33, pp. 77-80, 2005.

[18] S. Jansen and I. Wegener, "Evolutionary algorithms: how to cope with plateaus of constant fitness and when to reject strings of the same fitness," IEEE Trans. Evol. Comput., vol. 5, no. 6, pp. 589-599, 2001.

[19] J. He and X. Yao, "Drift analysis and average time complexity of evolutionary algorithms," Artif. Intell., vol. 127, no. 1, pp. 57-85, 2001.

[20] S. Droste, T. Jansen, and I. Wegener, "On the analysis of the $(1+1)$ evolutionary algorithm," Theor. Comput. Sci., vol. 276, no. 1-2, pp. 5181, 2002.

[21] J. He and X. Yao, "Towards an analytic framework for analysing the computation time of evolutionary algorithms," Artif. Intell., vol. 145, pp. $59-97,2003$

[22] F. Neumann, J. Reichel, and M. Skutella, "Computing minimum cuts by randomized search heuristics," Algorithmica, vol. 59, pp. 323-342, 2011.

[23] Y. Zhou, J. He, and Q. Nie, "A comparative runtime analysis of heuristic algorithms for satisfiability problems," Artif. Intell., vol. 173, pp. 240257, 2009.

[24] F. Neumann and I. Wegener, "Randomized local search, evolutionary algorithms, and the minimum spanning tree problem," Theor. Comput. Sci., vol. 378, pp. 32-40, 2007.

[25] F. Neumann, "Expected runtimes of evolutionary algorithms for the Eulerian cycle problem," Comput. Oper. Res., vol. 35, pp. 2750-759, 2008.

[26] A. Sutton and F. Neumann, "A parameterized runtime analysis of evolutionary algorithms for the euclidean traveling salesperson problem," in Proc. 26th Conference on Artificial Intelligence (AAAI'12), 2012, pp. 1105-1111.

[27] O. Giel and I. Wegener, "Evolutionary algorithms and the maximum matching problem," in Proc. 20th Annu. Symp. Theor. Aspects Comput. Sci. (STACS), LNCS vol. 2607, Berlin, Germany, 2003, pp. 415-426.
[28] P. Oliveto, J. He, and X. Yao, "Analysis of the (1+1)-EA for finding approximate solutions to vertex cover problems," IEEE Trans. Evol. Comput., vol. 13, no. 5, pp. 1006-1029, 2009.

[29] T. Friedrich, J. He, N. Hebbinghaus, F. Neumann, and C. Witt, "Approximating covering problems by randomized search heuristics using multi-objective models," Evol. Comput., vol. 18, no. 4, pp. 617-633, 2010.

[30] C. Witt, "Worst-case and average-case approximations by simple randomized search heuristics," in Proc. 22nd Annu. Symp. Theor. Aspects Comput. Sci. (STACS), LNCS vol. 3404. Stuttgart, Germany, Feb. 2005, pp. 44-56.

[31] F. Neumann and J. Reichel, "Approximating minimum multicuts by evolutionary multi-objective algorithms," in Proc. 10th Int. Conf. Parallel Problem Solving Nature (PPSN X), Dortmund, Germany, 2008, pp. 7281.

[32] Y. Yu, X. Yao, and Z. Zhou, "On the approximation ability of evolutionary optimization with application to minimum set cover," Artif. Intell., vol. 180-181, pp. 20-33, 2012

[33] F. Neumann and I. Wegener, "Minimum spanning trees made easier via multi-objective optimization," Natural Computing, vol. 5, no. 3, pp. 305-319, 2006.

[34] F. Neumann, "Expected runtimes of a simple evolutionary algorithm for the multi-objective minimum spanning tree problem," Eur. J. Oper. Res., vol. 181, pp. 1620-1629, 2007.

[35] C. Qian, Y. Yu, Z.-H. Zhou, "An analysis on recombination in multiobjective evolutionary optimization“, Artif. Intell., vol. 204, pp. 99-119, 2013.

[36] T. Brüggemann, J. Monnot, and G. Woeginger, "Local search for the minimum label spanning tree problem with bounded color classes," Oper. Res. Lett., vol. 31, pp. 195-201, 2003.

[37] O. Giel, "Expected runtimes of a simple multi-objective evolutionary algorithm," in Proc. IEEE Congr. Evol. Comput., 2003, pp. 1918-1925.

[38] M. Laumanns, L. Thiele, and E. Zitzler, "Running time analysis of multiobjective evolutionary algorithms on pseudo-boolean functions," IEEE Trans. Evol. Comput., vol. 8, no. 2, pp. 170-182, 2004.

[39] M. Mitzenmacher and E. Upfal, Propability and Computing. Cambridge: Cambridge University Press, 2005.

[40] T. Jansen, K. A. D. Jong, and I. Wegener, "On the choice of the offspring population size in evolutionary algorithms," Evol. Comput., vol. 13, no. 4, pp. 413-440, 2005

[41] T. Chen, J. He, G. Sun, G. Chen, and X. Yao, "A new approach to analyzing average time complexity of population-based evolutionary algorithms on unimodal problems," IEEE Trans. Syst., Man, Cybern., B Cybern., vol. 39, no. 5, pp. 1092-1106, 2009.

[42] T. Chen, K. Tang, G. Chen, and X. Yao, "A large population size can be unhelpful in evolutionary algorithms," Theor. Comput. Sci., vol. 436, no. 8, pp. 54-70, 2012.

[43] T. Jansen and I. Wegener, "Real royal road functions-where crossover provably is essential," Discrete Appl. Math., vol. 149, pp. 111-125, 2005.

[44] B. Doerr, E. Happ, C. Klein, "Crossover can provably be useful in evolutionary computation," Theor. Comput. Sci., vol. 425, pp. 17-33, 2012.

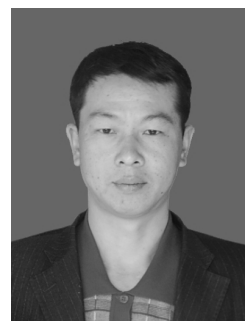

Xinsheng Lai received the M.Sc. degree in Computer Applied Technology from Guizhou University, Guiyang, China, in 2004, and he is now working towards the Ph.D. degree at South China University of Technology, Guangzhou, China. His main research interests include evolutionary computation, neural computation, and their applications in real world. 


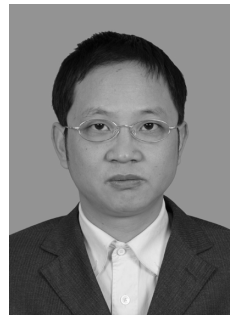

Yuren Zhou received the B. Sc. degree in the Mathematics from Peking University, Beijing, China, in 1988, the M. Sc. degree in the Mathematics from Wuhan University, Wuhan, China, in 1991, and the $\mathrm{Ph}$. D. in Computer Science from Wuhan University in 2003. He is currently a professor in School of Computer Science and Engineering, South China University of Technology, Guangzhou, China. His current research interests are focused on design and analysis of algorithms, evolutionary computation and data mining.

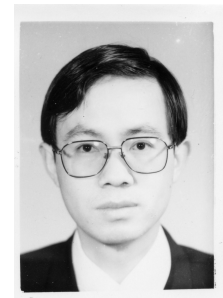

Jun He (M'06) received the B.S., M.Sc. degrees in mathematics and the Ph.D. degree in computer science all from Wuhan University, Wuhan, China, in 1989, 1992 and 1995, respectively.

$\mathrm{He}$ is currently a Senior Lecturer at Aberystwyth University, United Kingdom. His major research interests include evolutionary computation, global optimization and network security. He has published over 80 papers in these areas.

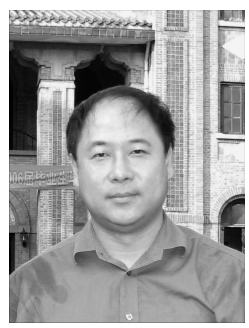

Jun Zhang (M'02-SM'08) received the Ph.D. degree in Electrical Engineering from the City University of Hong Kong, Kowloon, Hong Kong, in 2002. From 2003 to 2004, he was a Brain Korean 21 Post-Doctoral Fellow with the Department of Electrical Engineering and Computer Science, Korea Advanced Institute of Science and Technology, Daejeon, Korea. Since 2004, he has been with Sun Yat-Sen University, Guangzhou, China, where he is currently a Cheung Kong Professor with the School of Advanced Computing. He has authored seven research books and book chapters, and over 100 technical papers in his research areas. His current research interests include computational intelligence, cloud computing, high performance computing, data mining, wireless sensor networks, operations research, and power electronic circuits.

Dr. Zhang was a recipient of the China National Funds for Distinguished Young Scientists from the National Natural Science Foundation of China in 2011 and the First-Grade Award in Natural Science Research from the Ministry of Education, China, in 2009. He is currently an Associate Editor of the IEEE Transactions on Evolutionary Computation, the IEEE Transactions on Industrial Electronics, the IEEE Transactions on Cybernetics, and the IEEE Computational Intelligence Magazine. He is the Founding and Current Chair of the IEEE Guangzhou Subsection and IEEE Beijing (Guangzhou) Section Computational Intelligence Society Chapters. He is the Founding and Current Chair of the ACM Guangzhou Chapter. 